\title{
Comparative analysis of molecular mechanism of spinal cord injury with time based on bioinformatics data
}

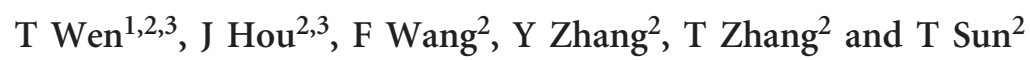

Objectives: This study was designed to explore the molecular mechanisms of spinal cord injury (SCI) with time.

Methods: The gene expression profile (GSE45006) including four non-injured spinal cord samples as sham-control group and 20 thoracic transected spinal cords samples as experimental group at different times was downloaded from Gene Expression Omnibus database. The time-course changes of the SCl-related differentially expressed genes (DEGs) were identified. In addition, time-series expression profile clusters of DEGs were obtained, followed by gene ontology (GO) and pathway enrichment analysis of the DEGs. Moreover, the transcriptional regulatory network was constructed.

Results: There were 1420, 492, 743, 568 and 533 DEGs respectively at d1, d3, w1, w2 and w8 compared with that of sham group. Importantly, 101 overlapped regulated DEGs were identified at five time points and 370 collaboratively regulated genes were identified in cluster 6. Significant functions of overlapped regulated DEGs were obtained including response to wounding and developmental process. In addition, the DEGs, such as CD14 molecule (CD14) and chemokine (C-C motif) ligand 2 (CCL2), were enriched mostly in the pathways related to tuberculosis, phagosome and NF-kappa B signaling pathway. From the transcriptional regulatory network, we identified some transription factors (TFs), including member of E26 transformation-specific (ETS) oncogene family (ELK1) and zinc finger and BTB domain containing 7A (Zbtb7a).

Conclusion: The DEGs related to immune response during $\mathrm{SCl}$ may provide underlying targets for treatment of SCl. Moreover, the TFs ZBTB7A and ELK1 and their target gene (dual specificity phosphatase 18 (DUSP18)) might be therapeutic targets for the treatment of $\mathrm{SCl}$.

Spinal Cord (2016) 54, 431-438; doi:10.1038/sc.2015.171; published online 27 October 2015

\section{INTRODUCTION}

Spinal cord injury (SCI) is a major cause of disability and a global medical challenge. ${ }^{1}$ The incidence of SCI is higher in China than other countries with about 60000 cases per year. ${ }^{2}$ The functional decline after SCI is caused by direct injury and secondary pathophysiological changes induced by the initial trauma. ${ }^{3}$ SCI not only lead to paraplegia or quadriplegia but also impacts on life expectancy and exacts financial burdens. ${ }^{4}$ Moreover, these patients are faced with increased risks of cardiovascular complications, osteoporosis and deep vein thrombosis. ${ }^{5}$ However, there are no fully restorative treatments for SCI as yet. ${ }^{6}$ Thus, it is a significant advance to find any novel therapeutic strategies to allow for major functional recovery for SCI.

The new techniques may have neuroprotective roles via acting as an anti-inflammatory treatment, providing the neuroprotective support for the remaining host cells and stimulating the regeneration of the central nervous system (CNS). ${ }^{7}$ However, regeneration of the damaged CNS in SCI is difficult. Recent studies have demonstrated that the ras homologue (Rho) signaling pathway has an important role of inhibiting neuronal growth. ${ }^{8}$ Parikh et al. ${ }^{9}$ have shown that transcriptional factor SMAD family member 1-dependent bone morphogenetic protein signaling regulates axonal growth in dorsal root ganglion neurons. Phospholipases $\mathrm{A}_{2}$ may represent a new and effective strategy to block multiple signaling pathways in SCI. ${ }^{10}$ Moreover, the expression of immediate early genes and cytokines
mRNAs has shown to be upregulated following SCI. ${ }^{11}$ However, the underlying mechanisms of SCI still remain fully unknown.

Generally, SCI is highly heterogeneous, and the therapeutic approach depends on the location, stage and time following SCI. The gene expression profile GSE45006 was provided by Chamankhah et al. ${ }^{12}$ who analyzed the temporal pattern of biological processes of gene ontology (GO) using the clip compression injury model in rats. Moreover, the gene expression profile GSE45006 has a wider time frame $(1,3,7,14$ and 56 days post injury) than other studies. However, the transcriptional regulatory network based on the coordinately regulated genes was not analyzed.

Therefore, the same expression profile GSE45006 was used in this study for screening the SCI-related differentially expressed genes (DEGs) with the time-course changes. In addition, time-series expression profile clusters of DEGs were obtained, followed by GO and pathway enrichment analysis of the DEGs. Moreover, the transcriptional regulatory network was constructed. We infer the results might give a deep insight into the molecular mechanisms and development of therapeutics strategy of SCI.

\section{MATERIALS AND METHODS}

Microarray data

The gene expression profile of GSE45006 was downloaded from the Gene Expression Omnibus database of the National Center of Biotechnology

\footnotetext{
${ }^{1}$ Chinese PLA Medical College, Beijing, China and ${ }^{2}$ Department of Orthopedics, Beijing Army General Hospital, Beijing, China

${ }^{3}$ These two authors contributed equally to this work.

Correspondence: Dr T Sun, Department of Orthopedics, Beijing Army General Hospital, No.5 Nanmencang, Dongcheng District, Beijing 100700, China.

E-mail: tianshengsuntss@163.com

Received 10 May 2015; revised 16 August 2015; accepted 1 September 2015; published online 27 October 2015
} 
Information (NCBI; http://www.ncbi.nlm.nih.gov/geo/), which was on the basis of GPL1355 platform of Affymetrix Rat Genome 2302.0 Array ([Rat230_2] (Affymetrix Inc., Santa Clara, CA, USA)). In the present study, there were 24 samples including 4 non-injured spinal cord samples as sham-control group and 20 thoracic transected spinal cords samples as experimental groups at 1day (d1, n=4),3 days (d3, n=4), 1 week (w1, $n=4), 2$ weeks (w2, $n=4)$ and 8 weeks (w8, $n=4$ ) post injury, respectively.

\section{Data preprocessing}

Robust multiarray average ${ }^{13}$ algorithm of the Partek Genomics Suite package (Partek, Inc, St Louis, MO, USA) was used to preprocess the gene expression profile of GSE45006. Specific steps were as follows: data in CEL format were converted into expression measures, followed by background correction, quantile normalization, $\log 2$ transformation, batch correction and probe summarization. Finally, the gene expression matrix was obtained. If there were several probes mapping to one Gene Symbol, the average was used to represent the expression level of this gene. There were 24447 probes in the raw data, and 12725 genes were obtained after data preprocessing.

\section{Identification of DEGs between SCI and control samples at five time points}

GSE45006 data include five experimental groups at different times (d1, d3, w1, w2 and w8) and 1 sham-control group (sham). Analysis of variance (ANOVA) and $t$-test were applied to analyze the DEGs between experimental groups and sham group. The Benjamini and Hochberg method ${ }^{14}$ was performed to adjust the raw $P$-value into the false discovery rate (FDR). The fold change of gene expression $(\log 2 \mathrm{FC})$ and the FDR were used to select the DEGs. FDR $<0.05$ and $\log 2 \mathrm{FCl}>2.5$ were chosen as the cutoff criteria.

\section{Time-series expression profile clustering}

BioLayout Express ${ }^{3 \mathrm{D}}$ (BioLayout) ${ }^{15}$ is an application designed for displaying network from biologically derived data. In the present study, all genes of 24 samples were inputted into BioLayout (http://www.biolayout.org/) to construct the gene-gene regulatory network. Pearson correlation coefficient was applied to determine the connective significance of a gene pair. The Pearson correlation coefficient $>0.85$ was chosen as the criterion for selecting co-regulated genes. Then, Markov clustering (MCL clustering) ${ }^{16}$ was applied to divide the large network into a certain number of subnet clusters. The inflation value parameter of the MCL algorithm is applied to control granularity of the cluster. In our study, the inflation value parameter is 4.0. Due to MCL clusters are arranged according to the size of the cluster. The clusters of more genes have a greater role in the coordinated entire regulatory network. Therefore, we selected the clusters of $>100$ genes as the important cooperative regulation clusters.

\section{GO functional enrichment analysis of the DEGs}

$\mathrm{GO}$ analysis has been used as functional enrichment studies of large-scale genes frequently. ${ }^{17}$ The biological significance of a set of genes between SCI and sham groups at different times can be assessed by GO enrichment analysis. The Biological Networks Gene Ontology tool (BiNGO) ${ }^{18}$ of a Cytoscape (Institute for Systems Biology, Seattle, WA, USA) plugin was used to assess overrepresentation of GO categories of overlapped regulated DEGs and collaboratively regulated genes in biological process. FDR $<0.05$ were chosen as the cutoff criterion.

\section{KEGG pathway enrichment analysis of the DEGs}

The Database for Annotation, Visualization and Integrated Discovery (DAVID) is a tool providing a comprehensive set of functional annotation. ${ }^{19}$ Kyoto Encyclopedia of Genes and Genomes (KEGG) is bioinformatics database which contains all kinds of biochemistry pathways. ${ }^{20}$ DAVID was used to identify DEGs associated pathways by calculating the $P$-value. The $P$-value $<0.05$ were selected as the cut-off criterion.

\section{Construction of transcriptional regulatory network}

Genomatix Software Suite (https://www.genomatix.de/) software package was preformed to predict the transcription factor binding site. First of all, Promoter sequences were extracted in Genomatix database using Gene2Promoter tool (Genomatrix, Munich, Germany). Then, transcription factor binding site was analyzed by MatInspector (https://www.genomatix.de/online_help/help_ matinspector/matinspector_help.html). ${ }^{21}$ Finally, A $P<0.05$ was chosen as the criterian of TF family and TF-target gene pairs. Cytoscape ${ }^{22}$ was performed to construct the network of TF-target genes.

\section{RESULTS}

\section{Data normalization}

The expression profile data behind and after normalization were shown in Figures $1 \mathrm{a}$ and $\mathrm{b}$.

\section{The DEGs between SCI and sham samples at five time points} Through analyzing the gene expression profile of the experimental groups at five time points after SCI and sham group, there were 1420, 492, 743, 568 and 533 DEGs respectively at d1, d3, w1, w2 and w8. As shown in Figure 2a, there were 1080 upregulated probes corresponded to 844 upregulates DEGs and 1088 downregulated probes relative to 576 DEGs. Figure $2 \mathrm{~b}$ depicts the Venn diagrams with overlapping regions exhibiting the number of overlapped genes showing the alterations at different time points and time point-specific genes. On the basis of the results, the $\mathrm{d} 1$ pattern of gene expression is more similar to sham as is evidenced by 1420 overlapped genes between $\mathrm{d} 1$ and sham group, compared with 743 between w1 and sham, 568 between w2 and sham, 533 between w8 and sham, and 492 between d3 and sham. Importantly, 101 overlapped regulated DEGs were identified at these five time points.

\section{Time-series expression profile clustering}

To further explore the changes of the DEGs expression levels at the five time points after SCI, we performed the cluster analysis. Based on the parameter of inflation for 'MCL clustering method', 2453 cooperative regulation clusters were obtained. Among these, there were 11 clusters including at least 100 genes (a total of 4853 genes, accounting for $48 \%$ of the entire network). Figure 3 showed a tread of all genes of distinct significant expression clusters. Cluster 1 included 1004 genes, and the expression level of these genes was the highest in sham group, followed by a decrease in gene expression with SCI and a fluctuation on the $\mathrm{d} 3$ post injury. Cluster 3 included 663 genes, and the expression level of these genes was the lowest in sham group while the highest on the $\mathrm{d} 1$ post injury followed by a decrease in gene expression with SCI. Cluster 6 contained 370 genes, and gene expression was further followed by escalation of gene expression at the $\mathrm{d} 1$ and $\mathrm{d} 3$, which peaked at $\mathrm{d} 3$ and decreased with time. In contrast, the expression of genes in cluster 6 was lower in sham group.

\section{GO functional pathway enrichment analysis}

The FDR $<0.05$ was chosen as threshold of functions of 101 overlapped regulated DEGs and 370 collaboratively regulated genes. Top 10 significantly enriched functions of 101 overlapped regulated DEGs were shown in Table 1 . The most remarkable function was response to wounding $(\mathrm{FDR}=3.89 \mathrm{E}-05)$. The other significant functions included system development (FDR $=7.24 \mathrm{E}-05)$, developmental process $(\mathrm{FDR}=7.65 \mathrm{E}-05)$. Moreover, 370 collaboratively regulated genes were enriched in nucleic acid metabolic process $(\mathrm{FDR}=9.05 \mathrm{E}-13)$, RNA metabolic process $(\mathrm{FDR}=8.77 \mathrm{E}-11)$ and cellular macromolecule metabolic process $(\mathrm{FDR}=1.00 \mathrm{E}-10$; Table 2). 

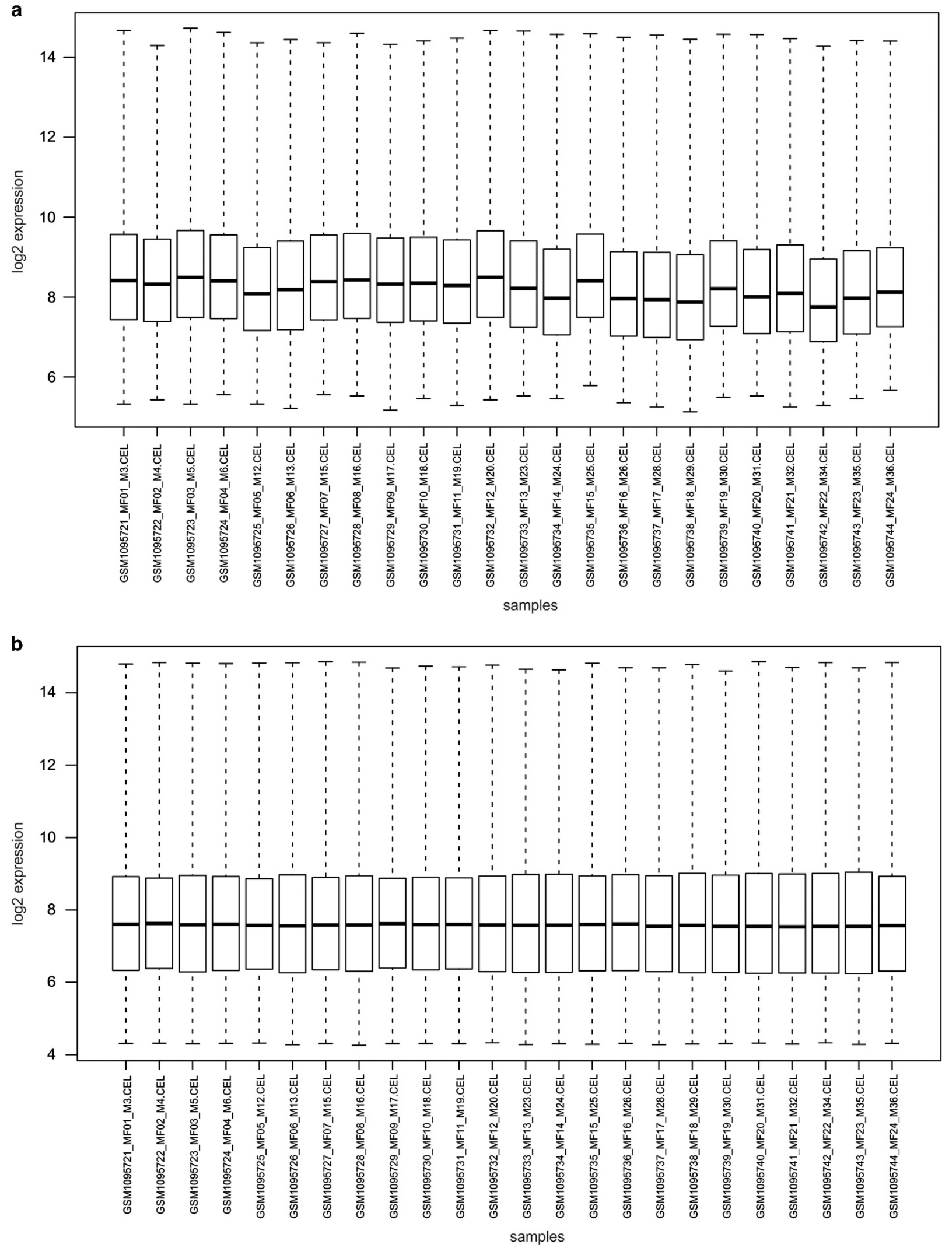

Figure 1 (a) Box plot of gene expressions in sham and spinal cord injury samples behind normalization. (b) Box plot of gene expressions in sham and spinal cord injury samples after normalization. The $x$ axis was samples whereas the $y$ axis was expression level of genes. The black line in the center was the median of expression value and the consistent distribution indicated a good standardization. 

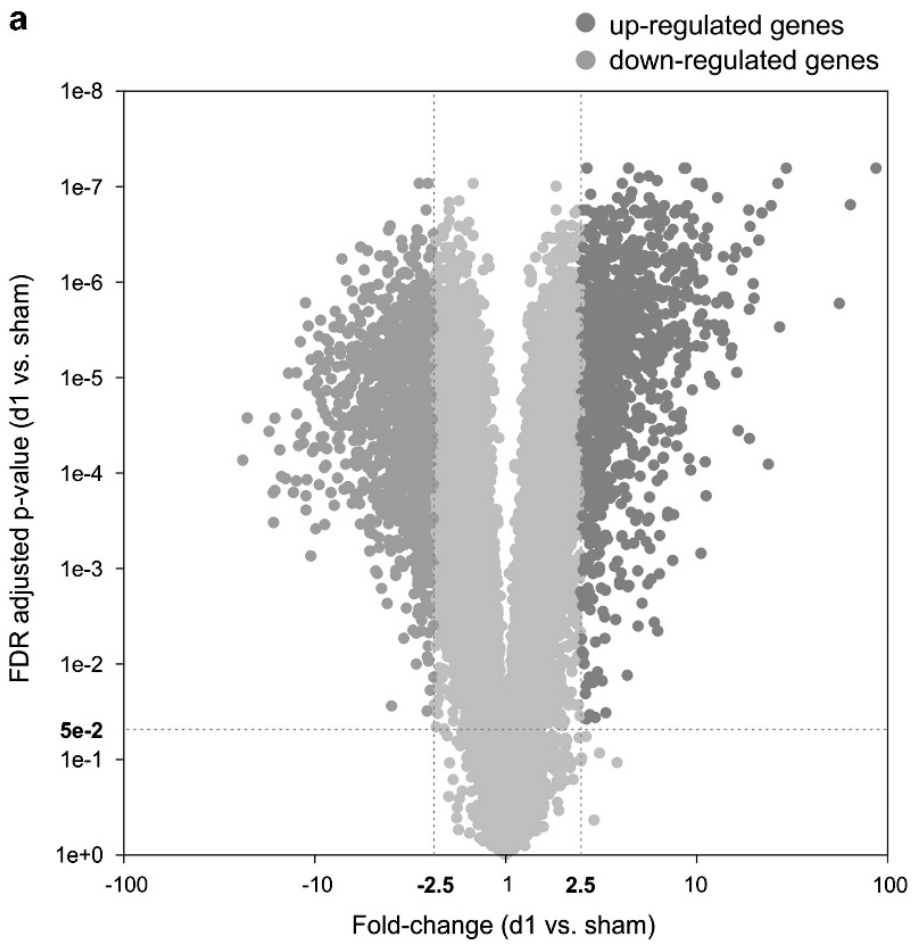

b

d1 vs. sham (1420)

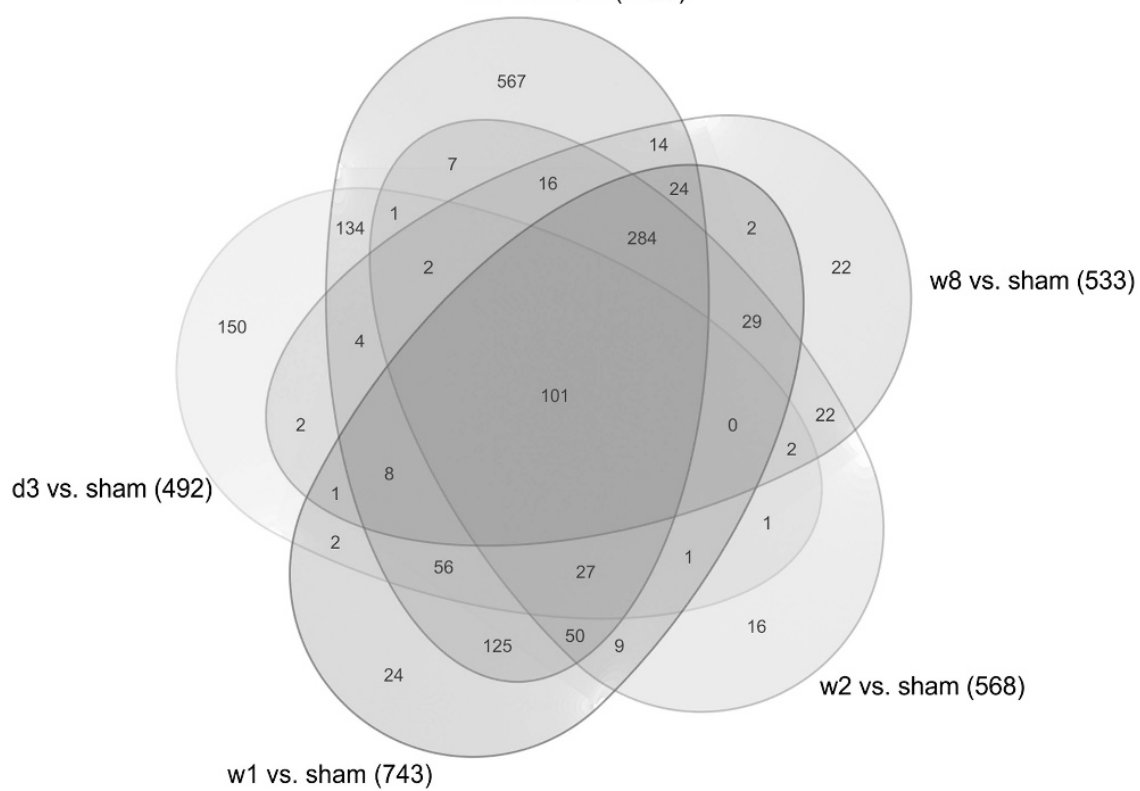

Figure 2 (a) Volcano plots of fold change values of all 2168 Probesets vs transformed (log2) and FDR at day 1 of experimental relative to sham group. Red points represent upregulated genes. Blue points represent downregulated genes. (b) Time-point gene set data analysis. Common and unique genes at each time point were examined a Venn diagram. Overlapping areas represent common genes between different time points. A full color version of this figure is available at the Spinal Cord journal online.

\section{KEGG pathway enrichment analysis}

The KEGG pathways of 101 overlapped regulated DEGs were enriched and shown in Table 3 . The results showed that the significantly enriched KEGG pathways were mainly on immune system related pathways, such as tuberculosis $(P=3.99 \mathrm{E}-06)$, nuclear factor kappa (NF-kappa) B signaling pathway $(P=0.000409062)$. The KEGG pathways of 370 collaboratively regulated genes were enriched and shown in Table 4 . The most remarkable pathways were RNA transport $(P=6.31 \mathrm{E}-10)$, ribosome biogenesis in eukaryotes $(P=1.80 \mathrm{E}-08)$ and spliceosome $(P=6.44 \mathrm{E}-08)$.

\section{Construction of transcriptional regulatory network}

Based on the transcription factor binding site of 370 genes in cluster 6 , the TFs and TF-target gene were identified. The transcriptional regulatory network of the member of ETS oncogene family (ELK1) and zinc finger and BTB domain containing 7A (Zbtb7a) and their 

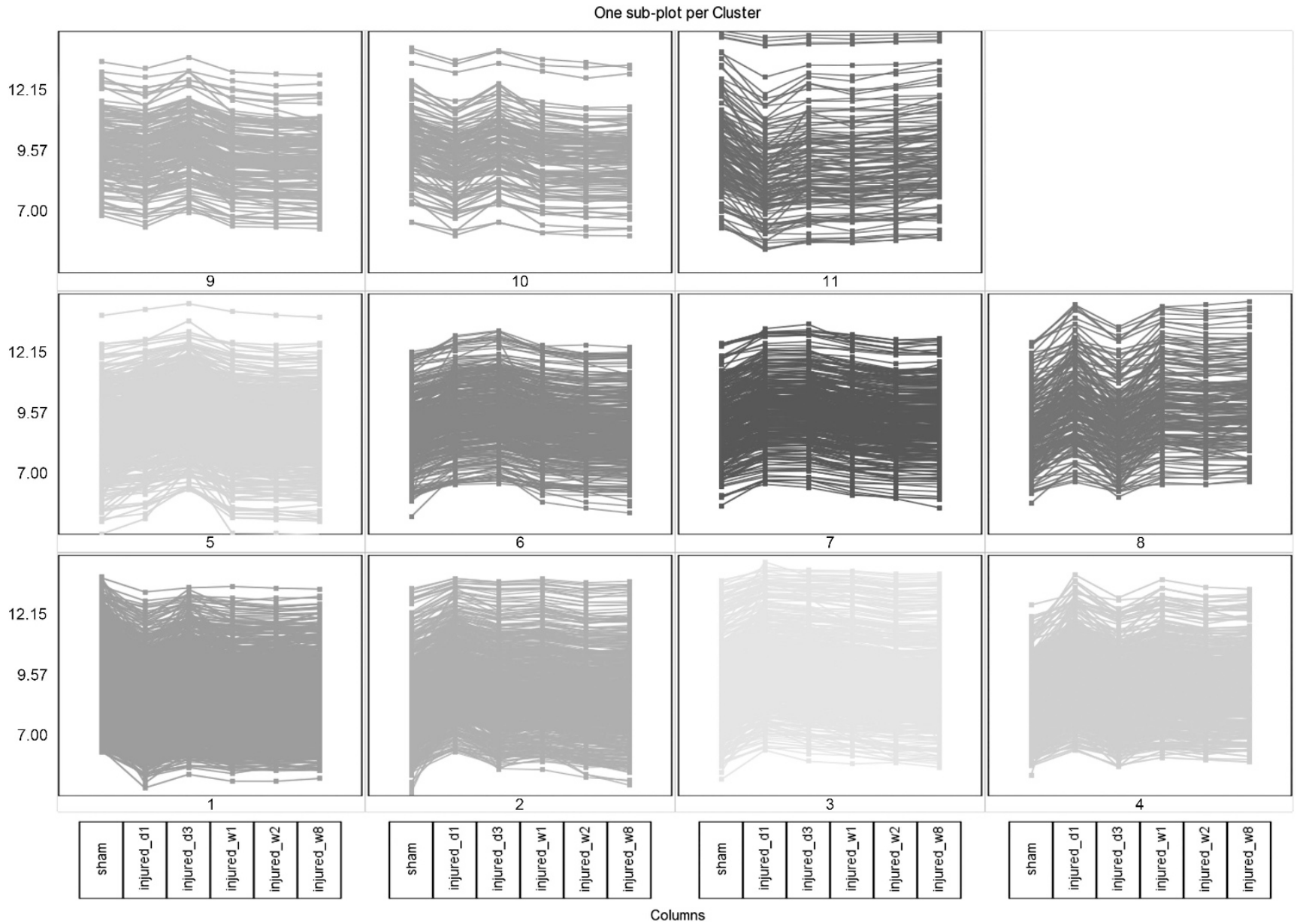

Figure 3 The trend chart of distinct significant expression profiles clustered by Markov clustering. The $x$ axis is samples (sham and experimental groups) whereas the $y$ axis is expression level of genes (the average is 0 , the s.d. is 1). Every curve represents the alteration of genes in samples between sham and experimental groups.

Table 1 The top 10 GO functional enrichment of 101 overlapped regulated genes at five time points of $\mathrm{SCl}$ in rats

\begin{tabular}{llccc}
\hline GO-ID & GO term & P-value & FDR & $\begin{array}{c}\text { Count of } \\
\text { genes }\end{array}$ \\
& & & & 14 \\
9611 & Response to wounding & $3.67 \mathrm{E}-08$ & $3.89 \mathrm{E}-05$ & 26 \\
48519 & Negative regulation of biological & $6.00 \mathrm{E}-08$ & $3.89 \mathrm{E}-05$ & \\
& process & & 24 \\
48523 & Negative regulation of cellular & $1.54 \mathrm{E}-07$ & $6.67 \mathrm{E}-05$ & 28 \\
& process & & & 28 \\
48731 & System development & $2.24 \mathrm{E}-07$ & $7.24 \mathrm{E}-05$ & 27 \\
32502 & Developmental process & $2.95 \mathrm{E}-07$ & $7.65 \mathrm{E}-05$ & 31 \\
48856 & Anatomical structure development & $3.67 \mathrm{E}-07$ & $7.91 \mathrm{E}-05$ & 28 \\
43066 & Negative regulation of apoptosis & $6.25 \mathrm{E}-07$ & $1.14 \mathrm{E}-04$ & 11 \\
43069 & Negative regulation of pro- & $7.05 \mathrm{E}-07$ & $1.14 \mathrm{E}-04$ & 11 \\
& grammed cell death & & & \\
60548 & Negative regulation of cell death & $1.03 \mathrm{E}-06$ & $1.48 \mathrm{E}-04$ & 11 \\
7275 & Multicellular organismal & $1.56 \mathrm{E}-06$ & $2.02 \mathrm{E}-04$ & 28 \\
& development & & & \\
\hline
\end{tabular}

Abbreviations: GO, gene ontology; FDR, false discovery rate; GO-ID, gene ontology identification; $\mathrm{SCl}$, spinal cord injury. targeted genes were shown in based in Figure 4. ELK1 regulated 200 target genes and $\mathrm{Zbtb} 7 \mathrm{a}$ regulated 212 target genes.

\section{DISCUSSION}

SCI is a fatal neurological disorder and there are no fully restorative treatments for SCI. ${ }^{6,23}$ In the current study, we investigated gene expression profile GSE45006 and explored the potential molecular mechanisms of SCI by bioinformatics methods. A total of 1420, 492, 743, 568 and 533 DEGs respectively at d1, d3, w1, w2 and w8 were screened. Importantly, 101 overlapped regulated DEGs were identified at these five time points. In addition, the overlapped regulated DEGs are enriched mostly in the pathways related to tuberculosis and NF-kappa B signaling pathway. From the transcriptional regulatory network, we identified some TFs in the DEGs, including ELK1 and Zbtb7a.

In the current study, some immune system-related pathways of the overlapped regulated DEGs were found. In addition, CD14 (CD14 molecule) is an overlapped regulated DEG, and the related pathways of it were tuberculosis, phagosome and NF-kappa B signaling pathway. Considerable evidence suggests the role of CD14 and innate immune responses in the CNS. ${ }^{24}$ The expression of CD14 is increased in spinal 
Table 2 The top 15 GO functional enrichment of 370 collaboratively regulated genes in cluster 6 at five time points of SCI in rats

\begin{tabular}{|c|c|c|c|c|}
\hline GO-ID & GO term & P-value & $F D R$ & Count of genes \\
\hline 16070 & RNA metabolic process & $1.13 \mathrm{E}-13$ & 8.77E-11 & 41 \\
\hline 6139 & Nucleobase, nucleoside, nucleotide and nucleic acid metabolic process & 2.87E-13 & 1.00E-10 & 61 \\
\hline 10467 & Gene expression & $3.24 \mathrm{E}-13$ & 1.00E-10 & 59 \\
\hline 34641 & Cellular nitrogen compound metabolic process & $1.21 \mathrm{E}-12$ & $3.11 \mathrm{E}-10$ & 66 \\
\hline 6807 & Nitrogen compound metabolic process & 7.38E-12 & $1.27 \mathrm{E}-09$ & 67 \\
\hline 44238 & Primary metabolic process & 2.85E-09 & 4.42E-07 & 130 \\
\hline 44237 & Cellular metabolic process & 4.33E-09 & $6.10 \mathrm{E}-07$ & 123 \\
\hline 22613 & Ribonucleoprotein complex biogenesis & $1.45 \mathrm{E}-08$ & $1.87 \mathrm{E}-06$ & 15 \\
\hline 42254 & Ribosome biogenesis & $4.80 \mathrm{E}-08$ & 5.73E-06 & 12 \\
\hline 9987 & Cellular process & 5.34E-08 & 5.91E-06 & 179 \\
\hline
\end{tabular}

Abbreviations: GO, gene ontology; FDR, false discovery rate; SCl, spinal cord injury.

Table 3 The KEGG pathways of the 101 overlapped regulated genes at five time points of $\mathrm{SCl}$ in rats

\begin{tabular}{|c|c|c|c|}
\hline Pathway name & $\begin{array}{c}\text { Enrichment } \\
\text { score }\end{array}$ & P-value & $\begin{array}{c}\% \text { genes in } \\
\text { pathway }\end{array}$ \\
\hline Tuberculosis & 12.4321 & 3.99E-06 & 6.2069 \\
\hline Staphylococcus aureus infection & 10.6453 & 2.38E-05 & 12.5 \\
\hline Phagosome & 8.46985 & 0.000209697 & 5 \\
\hline NF-kappa B signaling pathway & 7.80164 & 0.000409062 & 6.94444 \\
\hline Leishmaniasis & 6.39742 & 0.00166585 & 6.89655 \\
\hline Lysosome & 6.00666 & 0.00246229 & 4.6729 \\
\hline Systemic lupus erythematosus & 5.65346 & 0.00350537 & 5.6338 \\
\hline $\begin{array}{l}\text { Fc gamma R-mediated } \\
\text { phagocytosis }\end{array}$ & 5.31502 & 0.00491718 & 5.12821 \\
\hline $\begin{array}{l}\text { Transcriptional misregulation in } \\
\text { cancer }\end{array}$ & 4.97965 & 0.00687647 & 3.67647 \\
\hline $\begin{array}{l}\text { Chagas disease (American } \\
\text { trypanosomiasis) }\end{array}$ & 4.80996 & 0.00814821 & 4.44444 \\
\hline Osteoclast differentiation & 4.2171 & 0.0147413 & 3.73832 \\
\hline Pertussis & 3.96205 & 0.019024 & 4.6875 \\
\hline $\begin{array}{l}\text { Antigen processing and } \\
\text { presentation }\end{array}$ & 3.80206 & 0.0223247 & 4.41176 \\
\hline Rheumatoid arthritis & 3.72615 & 0.0240853 & 4.28571 \\
\hline ECM-receptor interaction & 3.68915 & 0.0249933 & 4.22535 \\
\hline $\begin{array}{l}\text { Toll-like receptor signaling } \\
\text { pathway }\end{array}$ & 3.65276 & 0.0259196 & 4.16667 \\
\hline Salmonella infection & 3.61695 & 0.0268644 & 4.10959 \\
\hline Influenza A & 3.55196 & 0.0286683 & 3.05344 \\
\hline TNF signaling pathway & 3.11322 & 0.0444575 & 3.37079 \\
\hline ABC transporters & 3.10598 & 0.0447806 & 5.26316 \\
\hline Chemokine signaling pathway & 3.02592 & 0.0485132 & 2.58065 \\
\hline
\end{tabular}

Abbreviations: ECM, extra cellular matrix; KEGG, Kyoto Encyclopedia of Genes and Genomes; $\mathrm{SCl}$, spinal cord injury; TNF, tumor necrosis factor.

cords of amyotrophic lateral sclerosis patients and mutant $\mathrm{Cu}^{2+} / \mathrm{Zn}^{2+}$ superoxide dismutase 1 mice. $^{25,26}$ Moreover, a former study revealed an up-regulation of molecules for leukocyte adhesion and aggregation as well as mediation of phagocytosis, containing CD14, CD44 and CD45 in dogs with spinal cord trauma. ${ }^{27}$ The increased expression of
Table 4 The KEGG pathways of the 370 collaboratively regulated genes in cluster 6 at five time points of $\mathrm{SCl}$ in rats

\begin{tabular}{lccc}
\hline Pathway name & $\begin{array}{c}\text { Enrichment } \\
\text { score }\end{array}$ & P-value & $\begin{array}{c}\text { \% genes in } \\
\text { pathway }\end{array}$ \\
\hline RNA transport & 21.1839 & $6.31 \mathrm{E}-10$ & 15 \\
Ribosome biogenesis in & 17.8313 & $1.80 \mathrm{E}-08$ & 19.4444 \\
eukaryotes & & & \\
Spliceosome & 16.558 & $6.44 \mathrm{E}-08$ & 14.2857 \\
RNA polymerase & 14.0947 & $7.56 \mathrm{E}-07$ & 29.6296 \\
Pyrimidine metabolism & 11.3857 & $1.14 \mathrm{E}-05$ & 13.4831 \\
Purine metabolism & 7.77512 & 0.000420058 & 8.84354 \\
& & & 13.9535 \\
Proteasome & 6.42598 & 0.00161895 & 11.6279 \\
Cytosolic DNA-sensing & 4.73549 & 0.00877813 & \\
pathway & & & 13.0435 \\
Protein export & 3.49725 & 0.0302806 & 9.7561 \\
Nucleotide excision repair & 3.39694 & 0.0334755 & 6.04027 \\
Protein processing in endo- & 3.36849 & 0.0344416 & \\
plasmic reticulum & & & \\
\hline Abbreviations: KEGG, Kyoto Encyclopedia of Genes and Genomes; SCl, spinal cord injury.
\end{tabular}

CD14 in SCI could be ascribed to microglial up-regulation. Other evidence indicate that $\mathrm{CD} 14$ / toll-like receptors may contribute to the inflammatory responses initiated by microglia. ${ }^{28}$ In addition, a previous study has demonstrated that pro-inflammatory gene expression, such as IL-1 $\alpha$, IL-1 $\beta$, CCL2 and TNF $\alpha$ mRNA are increased in post-SCI liver changes. In this study, CD14 and CCL2 (chemokine (C-C motif) ligand 2 ) were significantly un-regulated at five time points after SCI. Importantly, immune response contributes to maintain neurogenesis in the damaged CNS. ${ }^{29}$ Therefore, inhibiting the immune response and related genes are likely to be beneficial to recovery CNS after SCI functionally.

The TFs in cluster 6 at five time points after SCI were found involved in ZBTB7A and ELK1. ZBTB7A is characterized by a peculiar protein structure in humans. ${ }^{30}$ The $Z b t b 7 a$ gene encodes for leukemia/ lymphoma-related factor (LRF) protein. LRF regulates lymphoid lineage fate decisions via decreasing the T-cell inductive effect of Notch1 signaling, and thereby favoring B-cell development. ${ }^{31}$ In 


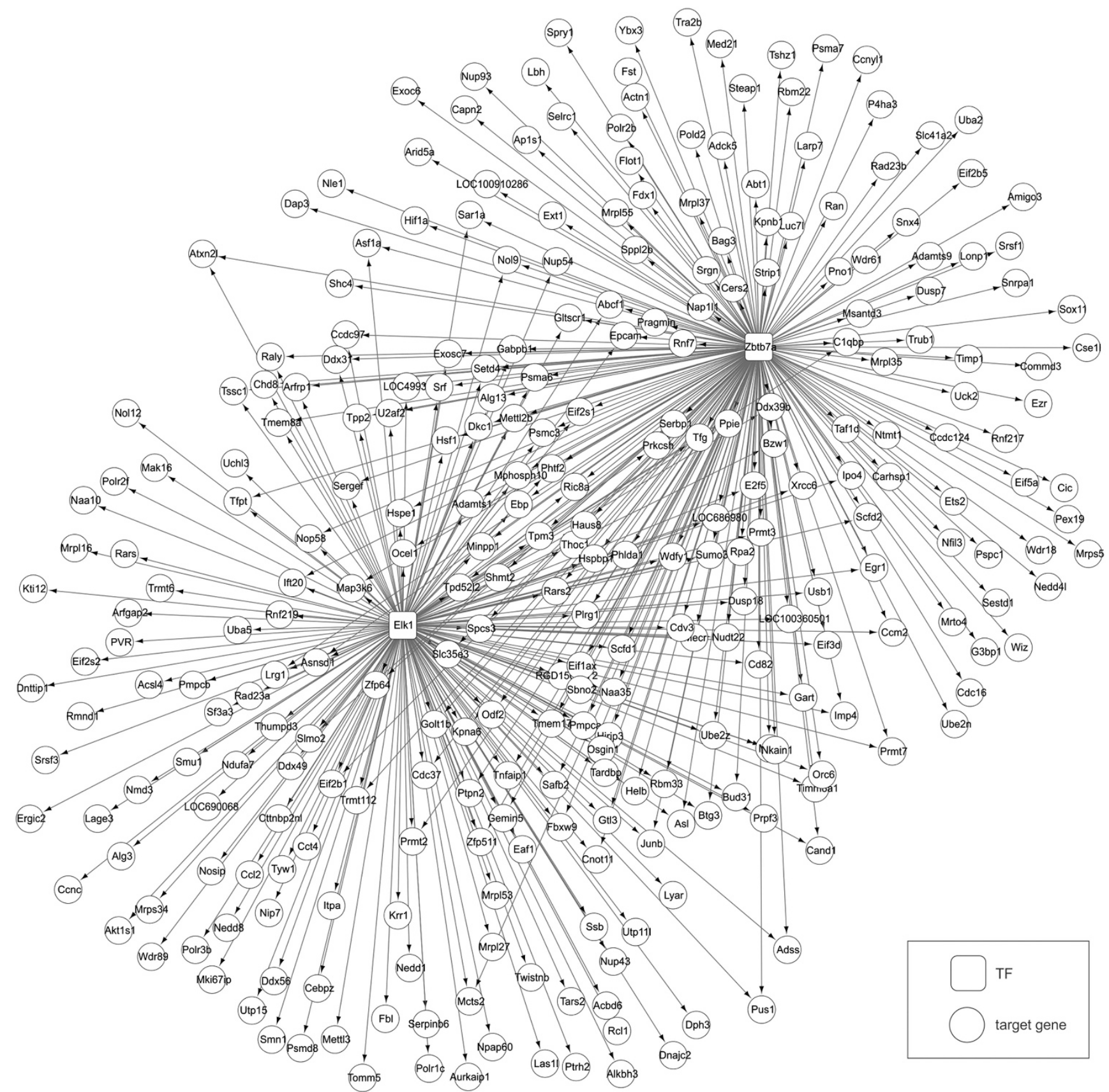

Figure 4 The transcriptional regulatory network of transcriptional factors ELK1 and Zbtb7a and their targeted genes. Box nodes represent transcriptional factors; Circle nodes represent target genes.

addition, a former study has demonstrated that LRF is expressed in the developing CNS and has a critical role of oligodendrocyte differentiation during the development of myelination in the CNS. ${ }^{32}$ As we all know, regulated by neuro-inflammation, oligoden-drocytes could deteriorate the development of SCI by causing axonal conduction abnormal. ${ }^{33}$ The ability of neurons to regenerate their axons after nerve injury requires extensive alterations in gene transcription. Activation of ELK-1 was observed $8 \mathrm{~h}$ after excitotoxic SCI. ${ }^{34}$ Extracellular signal-regulated kinases (ERKs), the mitogen-activated protein kinase family members, respond to growth factors by phosphorylating the transcription factor ELK1. ${ }^{35}$ The ERK-ELK1 pathway might contribute to the regulation of axonal growth. ${ }^{36}$
Dual specificity phosphatase 18 (DUSP18), a member of DUSPs, is a negative regulator of mitogen-activated protein kinases that functions by dephosphorylating. ${ }^{37}$ In addition, DUSP18 mRNA increases rapidly within $15 \mathrm{~min}$ in human embryonic kidney-293 cells treated with serum, ${ }^{38}$ activity accordant with that of an early-response gene. In the current study, DUSP18 was target gene of ELK1. Moreover, DUSP18 was further followed by escalation of gene expression at the $\mathrm{d} 1$ and $\mathrm{d} 3$, which peaked at $\mathrm{d} 3$ and decreased with time. For this reason, DUSP18 may participate in the early regenerative response. Therefore, we infer that TFs ZBTB7A and ELK1 and their target genes promote the development of damaged nervous system via activating oligodendrocyte differentiation. 
In conclusion, we have identified some related genes of SCI using bioinformatics analysis of gene expression. The immune system-related pathways of the overlapped regulated DEGs were enriched and the related genes such as CD14 and CCL2 were highlighted. Moreover, some TFs including ELK1 and Zbtb7a were identified. Importantly, DUSP18 may participate in the early regenerative response. However, further research is necessary to verify based on animal experiments because the study was based on microarray data.

\section{DATA ARCHIVING}

There were no data to deposit.

\section{CONFLICT OF INTEREST}

The authors declare no conflict of interest.

1 Profyris C, Cheema SS, Zang D, Azari MF, Boyle K, Petratos S. Degenerative and regenerative mechanisms governing spinal cord injury. Neurobiol Dis 2004; 15: 415-436.

2 Qiu J. China Spinal Cord Injury Network: changes from within. Lancet Neurol 2009; 8 : 606-607.

3 Carlson GD, Gorden C. Current developments in spinal cord injury research. Spine J 2002; 2: 116-128.

4 Oyinbo CA. Secondary injury mechanisms in traumatic spinal cord injury: a nugget of this multiply cascade. Acta Neurobiol Exp (Wars) 2011; 71: 281-299.

5 Vawda R, Fehlings MG. Mesenchymal cells in the treatment of spinal cord injury: current \& future perspectives. Curr Stem Cell Res Ther 2013; 8: 25-38.

6 Thuret S, Moon LD, Gage FH. Therapeutic interventions after spinal cord injury. Nat Rev Neurosci 2006; 7: 628-643.

7 Shi B, Ding J, Liu Y, Zhuang X, Zhuang X, Chen X et al. ERK1/2 pathway-mediated differentiation of IGF-1-transfected spinal cord-derived neural stem cells into oligodendrocytes. PLOS ONE 2014; 9: e106038.

8 Ruchhoeft ML, Ohnuma S, McNeill L, Holt CE, Harris WA. The neuronal architecture of Xenopus retinal ganglion cells is sculpted by rho-family GTPases in vivo. J Neurosci 1999; 19: 8454-8463.

9 Parikh P, Hao Y, Hosseinkhani M, Patil SB, Huntley GW, Tessier-Lavigne M et al. Regeneration of axons in injured spinal cord by activation of bone morphogenetic protein/Smad1 signaling pathway in adult neurons. Proc Natl Acad Sci 2011; 108: E99-E107.

10 Liu N-K, Xu X-M. Phospholipase A2 and its molecular mechanism after spinal cord injury. Mol Neurobiol 2010; 41: 197-205.

11 Hayashi M, Ueyama T, Nemoto K, Tamaki T, Senba E. Sequential mRNA expression for immediate early genes, cytokines, and neurotrophins in spinal cord injury. J Neurotrauma 2000: 17: 203-218.

12 Chamankhah M, Eftekharpour E, Karimi-Abdolrezaee S, Boutros PC, San-Marina S, Fehlings MG. Genome-wide gene expression profiling of stress response in a spinal cord clip compression injury model. BMC Genomics 2013; 14: 1-25.

13 Irizarry RA, Hobbs B, Collin F, Beazer-Barclay YD, Antonellis KJ, Scherf $U$ et al. Exploration, normalization, and summaries of high density oligonucleotide array probe level data. Biostatistics 2003; 4: 249-264.

14 Benjamini Y, Drai D, Elmer G, Kafkafi N, Golani I. Controlling the false discovery rate in behavior genetics research. Behav Brain Res 2001; 125: 279-284.
15 Theocharidis A, Van Dongen S, Enright AJ, Freeman TC. Network visualization and analysis of gene expression data using BioLayout Express3D. Nat Protoc 2009; 4: $1535-1550$.

16 Enright AJ, Van Dongen S, Ouzounis CA. An efficient algorithm for large-scale detection of protein families. Nucleic Acids Res 2002; 30: 1575-1584.

17 Ashburner M, Ball CA, Blake JA, Botstein D, Butler H, Cherry JM et al. Gene ontology: tool for the unification of biology. The Gene Ontology Consortium. Nat Genet 2000; 25: 25-29.

18 Maere S, Heymans K, Kuiper M. BiNGO: a Cytoscape plugin to assess overrepresentation of gene ontology categories in biological networks. Bioinformatics 2005; 21: 3448-3449.

19 Huang da W, Sherman BT, Lempicki RA. Systematic and integrative analysis of large gene lists using DAVID bioinformatics resources. Nat Protoc 2009; 4: 44-57.

20 Kanehisa M, Goto S. KEGG: kyoto encyclopedia of genes and genomes. Nucleic Acids Res 2000; 28: 27-30.

21 Cartharius K, Frech K, Grote K, Klocke B, Haltmeier M, Klingenhoff A et al. MatInspector and beyond: promoter analysis based on transcription factor binding sites. Bioinformatics 2005; 21: 2933-2942.

$22 \mathrm{Kohl} \mathrm{M}$, Wiese S, Warscheid BCytoscape: software for visualization and analysis of biological networks In: Data Mining in Proteomics. Humana Press, 2011, pp 291-303.

23 La Spada A, Ranum LP. Molecular genetic advances in neurological disease: special review issue. Hum Mol Genet 2010; 19: R1-R3.

24 Cao L, Tanga FY, DeLeo JA. The contributing role of CD14 in toll-like receptor 4 dependent neuropathic pain. Neuroscience 2009; 158: 896-903.

25 Henkel JS, Engelhardt JI, Siklós L, Simpson EP, Kim SH, Pan T et al. Presence of dendritic cells, MCP-1, and activated microglia/macrophages in amyotrophic lateral sclerosis spinal cord tissue. Ann Neurol 2004; 55: 221-235.

26 Henkel JS, Beers DR, Siklós L, Appel SH. The chemokine MCP-1 and the dendritic and myeloid cells it attracts are increased in the MSOD1 mouse model of ALS. Mol Cell Neurosci 2006; 31: 427-437.

27 Boekhoff TMA, Ensinger E-M, Carlson R, Bock P, Baumgärtner W, Rohn K et al. Microglial contribution to secondary injury evaluated in a large animal model of human spinal cord trauma. J Neurotrauma 2012; 29: 1000-1011.

28 Olson JK, Miller SD. Microglia initiate central nervous system innate and adaptive immune responses through multiple TLRs. J Immunol 2004; 173: 3916-3924.

29 Ziv Y, Avidan H, Pluchino S, Martino G, Schwartz M. Synergy between immune cells and adult neural stem/progenitor cells promotes functional recovery from spinal cord injury. Proc Natl Acad Sci 2006; 103: 13174-13179.

30 Kelly KF, Daniel JM. POZ for effect-POZ-ZF transcription factors in cancer and development. Trends Cell Biol 2006; 16: 578-587.

31 Maeda T, Merghoub T, Hobbs RM, Dong L, Maeda M, Zakrzewski J et al. Regulation of $B$ versus T lymphoid lineage fate decision by the proto-oncogene LRF. Science 2007; 316: 860-866.

32 Lunardi A, Guarnerio J, Wang G, Maeda T, Pandolfi PP. Role of LRF/Pokemon in lineage fate decisions. Blood 2013; 121: 2845-2853.

33 Mattson MP, Camandola S. NF-kB in neuronal plasticity and neurodegenerative disorders. J Clin Invest 2001; 107: 247-254.

34 Yu C-G, Yezierski RP. Activation of the ERK1/2 signaling cascade by excitotoxic spinal cord injury. Mol Brain Res 2005; 138: 244-255.

35 Drewett V, Muller S, Goodall J, Shaw PE. Dimer formation by ternary complex factor ELK-1. J Biol Chem 2000; 275: 1757-1762.

36 Robinson MJ, Stippec SA, Goldsmith E, White MA, Cobb MH. A constitutively active and nuclear form of the MAP kinase ERK2 is sufficient for neurite outgrowth and cell transformation. Curr Biol 1998; 8: 1141-1152.

37 Owens DM, Keyse SM. Differential regulation of MAP kinase signalling by dualspecificity protein phosphatases. Oncogene 2007; 26: 3203-3213.

38 Wu Q, Huang S, Sun Y, Gu S, Lu F, Dai J et al. Dual specificity phosphotase 18, interacting with SAPK, dephosphorylates SAPK and inhibits SAPK/JNK signal pathway in vivo. Front Biosci 2005; 11: 2714-2724. 